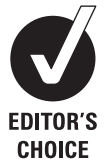

'Department of Endocrinology and Metabolism, Peninsula Medical School, Plymouth, UK ${ }^{2}$ Department of Mathematics and Statistics, University of Plymouth, Plymouth, UK

\section{Correspondence to}

Brad S Metcalf, Department

of Endocrinology

and Metabolism,

Peninsula Medical School University Medicine, Level 7, Derriford Hospital,

Plymouth PL6 8DH, UK; brad.metcalf@phnt.swest. nhs.uk

Accepted 26 April 2010 Published Online First 23 June 2010

\title{
Fatness leads to inactivity, but inactivity does not lead to fatness: a longitudinal study in children (EarlyBird 45)
}

\author{
B S Metcalf, J Hosking, ${ }^{1}$ A N Jeffery, ${ }^{1}$ L D Voss, ${ }^{1}$ W Henley, ${ }^{2}$ T J Wilkin ${ }^{1}$
}

\begin{abstract}
Objective To establish in children whether inactivity is the cause of fatness or fatness the cause of inactivity.

Design A non-intervention prospective cohort study examining children annually from 7 to 10 years. Baseline versus change to follow-up associations were used to examine the direction of causality.
\end{abstract}

Setting Plymouth, England.

Participants 202 children (53\% boys, 25\% overweight/ obese) recruited from 40 Plymouth primary schools as part of the EarlyBird study.

Main outcome measures Physical activity (PA) was measured using Actigraph accelerometers. The children wore the accelerometers for 7 consecutive days at each annual time point. Two components of PA were analysed: the total volume of PA and the time spent at moderate and vigorous intensities. Body fat per cent $(\mathrm{BF} \%)$ was measured annually by dual energy $x$ ray absorptiometry.

Results $\mathrm{BF} \%$ was predictive of changes in PA over the following 3 years, but PA levels were not predictive of subsequent changes in BF\% over the same follow-up period. Accordingly, a 10\% higher $\mathrm{BF} \%$ at age 7 years predicted a relative decrease in daily moderate and vigorous intensities of 4 min from age 7 to 10 years $(r=-0.17, p=0.02)$, yet more PA at 7 years did not predict a relative decrease in $\mathrm{BF} \%$ between 7 and 10 years $(r=-0.01, p=0.8)$.

Conclusions Physical inactivity appears to be the result of fatness rather than its cause. This reverse causality may explain why attempts to tackle childhood obesity by promoting PA have been largely unsuccessful.

The prevalence of childhood overweight/obesity is increasing annually in many developed and developing countries, ${ }^{1}$ reaching approximately $30 \%$ in the UK and the USA by $2004 .{ }^{23}$ Childhood overweight/obesity is of concern because it may be a precursor to serious health complications (eg, diabetes, cardiovascular disease, metabolic disturbance) later in life. ${ }^{4}$ Type 2 diabetes, which a generation ago seldom emerged before middle age, is increasingly a disease of adolescence and childhood. ${ }^{5}$

The cause of obesity is multifactorial but results, in the main, from a chronic imbalance between energy intake and energy expenditure (basal/ resting, physical activity (PA) and thermogenic response to feeding). PA accounts for 25\%-35\% of total energy expenditure in children ${ }^{6}$ and is deemed important because it is potentially modifiable. The widely held belief is that inactivity

\section{What is already known on this topic}

- It is widely believed from cross-sectional studies that childhood obesity is caused by physical inactivity.

- Direction of causality cannot be inferred from cross-sectional associations, their findings could equally represent obesity leading to physical inactivity.

- Public health and school-based interventions designed to make children more active rarely succeed in reducing obesity.

\section{What this study adds}

- Physical inactivity is the result rather than the cause of obesity.

- The relationship between fatness and PA is dominated by the impact of fatness on activity and not at all by activity on fatness.

- This reverse causality may explain why PA interventions so often fail to prevent excess weight gain in children.

leads to fatness, and this is reflected in a series of public health initiatives aimed at making children more active. The UK government has recently published guidelines on PA in preschool and school-aged children ${ }^{7}$ and is currently promoting Change4Life, a campaign that encourages families to adopt a more active lifestyle. ${ }^{8}$

Although there is overwhelming evidence that PA and obesity are linked, observational and experimental data do not always agree. Thus, two large and well-established European observational studies reported inverse associations $(r \approx-0.20)$ between objectively measured PA and body fat per cent (BF\%), ${ }^{10}$ whereas only 3 of 11 intervention trials reviewed by Wareham and colleagues ${ }^{11}$ showed even a modest impact of PA on body composition. The authors of the observational studies both conceded that their crosssectional design could not infer the direction of causality, yet both interpreted their findings as "inactivity leads to fatness". One explanation for the inconsistency between observational and experimental findings could be reverse causality. 
The cross-sectional correlations could equally well mean that fatness leads to inactivity, in which case PA intervention would not be expected to affect body mass.

Cause must precede effect, and longitudinal studies that measure fatness and activity levels at baseline and long-term follow-up can use the rule of temporality to investigate the dominant direction of causality. Four studies have carried out this kind of analysis in adults, ${ }^{12-15}$ but none has done so in children. All four adult studies reported a significant inverse association between baseline fatness and follow-up PA but not between baseline PA and follow-up fatness, suggesting that fatness leads to inactivity but that inactivity does not lead to fatness. The aim of the present study was to use the rule of temporality to elucidate the dominant direction of causality between objective measures of $\mathrm{PA}$ and $\mathrm{BF} \%$ in children.

\section{METHODS}

\section{Design, setting and participants}

The EarlyBird study is a non-intervention prospective cohort study investigating the factors that lead to childhood obesity and its associated metabolic disturbances. Some 307 healthy children (55\% boys, 98\% Caucasian) were recruited at school entry (aged 5 years) between January 2000 and January 2001 from 54 Plymouth primary schools, randomised to ensure a socio-economic mix representative of the city and of the UK in general (index of multiple deprivation 2004 score: EarlyBird cohort 26.1, Plymouth 26.3 and England 21.7 with cities ranging from 8 to 45). ${ }^{16}$ The study's rationale, recruitment procedures and protocol have been reported in detail elsewhere. ${ }^{17}$ The exclusion criteria included diabetes, pathologic conditions likely to affect growth or body composition, moderate or severe physical disability and long-term use of oral steroids. The cohort is measured annually, and this report covers four annual time points from age 7 years (when BF\% was first measured objectively) to age 10 years. The mean age at each time point is narrow (SD 3 months), deemed important for the resolution of age-related events, and the follow-up interval was 1.0 year (SD 1 month). Local research ethics committee approval was obtained in 1999.

\section{Measures}

\section{Physical activity}

PA was measured objectively on four annual occasions using Actigraph accelerometers (formerly MTI/CSA, Fort Walton Beach, Florida, USA). Children were asked to wear the accelerometers for 7 consecutive days (5 school days and 2 weekend days) at each annual time point, and only recordings that captured at least 5 days (including 1 weekend day) were used. The Actigraph records the intensity of movement every onetenth of a second, and for this study, the counts were collected into epochs of $1 \mathrm{~min}$ and stored against clock time. The parents were asked to record periods when their child removed the accelerometer during waking time, so that false periods of inactivity could be identified. False periods were replaced with the mean accelerometer counts recorded at the same clock time on the other days. Total PA (counts per week) and time spent in moderate and vigorous PA (MVPA, minutes per day) were analysed. Actigraphs have been shown to correlate well with free-living measures of energy expenditure in children ( $r=0.70$ independent of body weight ${ }^{18}$; $r=0.92$ with body weight ${ }^{19}$ ), and their technical reproducibility is impressive (between-Actigraph coefficient of variability 5\%; withinActigraph coefficient of variability $<2 \%) .{ }^{20}$ The sensitivity of each accelerometer was measured under controlled conditions by a motorised turntable. ${ }^{20}$ Seasonality was measured on a continuous scale by the number of relevant daylight hours (between 08:00 and 21:00) specific to Plymouth for the week the accelerometer was worn. ${ }^{21}$

\section{Body fat}

Whole BF\% was measured by dual energy $x$ ray absorptiometry (DEXA; Lunar Expert, Lunar, Madison, New York, USA), considered to be a criterion method for measuring body composition. ${ }^{22}$ Body mass index (BMI, kilogram per meter squared) and waist circumference (WC) were also measured and expressed as age- and sex-specific SD scores (BMI-SDS and WC-SDS) using the 1990 UK reference data.

\section{Sample size}

Of the 278, 269, 265 and 259 children who attended the appointments at age 7, 8, 9 and 10 years, respectively, 238, 230, 229 and 225, respectively, had measures of PA and BF\%. To maximise sample comparability, this report is based on the 202 children (107 boys and 95 girls) for whom measures of PA and $\mathrm{BF} \%$ were obtained at all four time points.

\section{STATISTICS}

All data analyses were carried out using SPSS V.15. Cohort characteristics were summarised by the means and SD for each sex at each annual time point, except for BF\% that was positively skewed and expressed as the median and the interquartile range. Each annual sample of PA (total PA and MVPA) was adjusted for seasonality and the sensitivity of the accelerometers using the respective regression coefficients obtained when modelling PA.

Multiple linear regression modelling was performed to quantify the association between PA and the measures of $\mathrm{BF} \%$ cross sectionally at each annual time point, 7, 8, 9 and 10 years, adjusted for age and sex (eg, $\left.\mathrm{PA}_{7 \mathrm{y}}=\mathrm{Sex}+\mathrm{Age}_{7 \mathrm{y}}+\mathrm{BF} \%_{7 \mathrm{y}}\right)$. The residuals generated from the models did not violate the assumptions of multiple linear regression modelling. The same method was used to establish the time-lagged association of $\mathrm{PA}$ on future $\mathrm{BF} \%$ measured 1,2 and 3 years later and, the reverse, $\mathrm{BF} \%$ on future $\mathrm{PA}$ measured 1,2 and 3 years later (eg, $\left.\mathrm{PA}_{10 \mathrm{y}}=\mathrm{Sex}+\mathrm{Age}_{7 \mathrm{y}}+\mathrm{BF} \%_{7 y}\right) \cdot{ }^{23}$

Changes in $\mathrm{PA}$ and $\mathrm{BF} \%$ were calculated for each child over each 1-, 2- and 3-year period. Multiple regression modelling was then performed to quantify the association between the predictor at a single time point and the change in the outcome variable from that time point to a 1-, 2- or 3-year follow-up. These models were adjusted for the outcome measure at the earlier time-point (eg, $\left.\mathrm{PA}_{10 \mathrm{y}}-\mathrm{PA}_{7 \mathrm{y}}=\mathrm{Sex}+\mathrm{Age}_{7 \mathrm{y}}+\mathrm{PA}_{7 \mathrm{y}}+\mathrm{BF} \%_{7 y}\right){ }^{23}$

\section{RESULTS \\ Trends}

Girls had higher BMI-SDS, WC-SDS and BF\% than boys and were less physically active (table 1). In both sexes, BMI-SDS, WC-SDS and BF\% increased with age and PA (total and MVPA) decreased with age.

\section{Associations (cross sectional)}

Whereas measures of fatness and PA differed between the sexes, the strength of association between the variables did not (sex interactions: all p>0.2). Consequently, all associations (cross sectional, time lagged and change in outcome) were derived from boys and girls together with adjustment for sex. The cross-sectional correlations between $\mathrm{BF} \%$ and $\mathrm{PA}$ were 
similar for total PA ( $r=-0.18$ to -0.23 ) and MVPA ( $r=-0.20$ to -0.25 ) at all ages (tables 2 and 3 ). The cross-sectional correlations were slightly lower for BMI-SDS versus PA (eg, MVPA: $\mathrm{r}=-0.12$ to -0.18$)$ and WC-SDS versus PA (eg, MVPA: $r=-0.10$ to -0.22$)$.

\section{Associations (time lagged)}

Simple time-lagged correlations of $\mathrm{BF} \%$ versus future PA were slightly, though not significantly, stronger than the correlations for $\mathrm{PA}$ versus future $\mathrm{BF} \%$ when the time lag was 1 year (eg, MVPA: $r=-0.27$ vs -0.19$), 2$ years $(r=-0.22$ vs -0.20$)$ and

Table 1 Summary characteristics by age and sex (mean (SD))

\begin{tabular}{|c|c|c|c|c|c|}
\hline \multirow[b]{2}{*}{ Sex } & \multirow[b]{2}{*}{ Variable } & \multicolumn{4}{|l|}{ Visit } \\
\hline & & 7 years & 8 years & 9 years & 10 years \\
\hline \multirow[t]{6}{*}{ Boys $(n=107)$} & Age (years) & $6.89(0.26)$ & $7.85(0.28)$ & $8.87(0.28)$ & $9.91(0.29)$ \\
\hline & BMI (SD score) & $0.21(1.09)$ & $0.30(1.14)$ & $0.38(1.13)$ & $0.44(1.14)$ \\
\hline & WC (SD score) & $0.19(1.04)$ & $0.36(1.09)$ & $0.45(1.10)$ & $0.58(1.06)$ \\
\hline & Body fat† (\%) & $13.0(7.4)$ & $13.6(8.8)$ & $16.0(12.0)$ & $18.8(15.3)$ \\
\hline & Total PA (counts $\times 10^{5} /$ week) & $38.7(8.3)$ & $38.7(8.4)$ & $37.8(8.0)$ & $35.8(9.4)$ \\
\hline & MVPA (min/day) & $56.9(22.6)$ & $57.3(21.5)$ & $56.5(19.6)$ & $52.3(24.1)$ \\
\hline \multirow[t]{6}{*}{ Girls $(n=95)$} & Age (years) & $6.88(0.25)$ & $7.82(0.29)$ & $8.84(0.29)$ & $9.87(0.28)$ \\
\hline & BMI (SD score) & $0.58(1.05)$ & $0.61(1.12)$ & $0.70(1.10)$ & $0.73(1.17)$ \\
\hline & WC (SD score) & $0.48(1.14)$ & $0.53(1.20)$ & $0.74(1.23)$ & $0.87(1.27)$ \\
\hline & Body fat $†(\%)$ & $20.0(11.9)$ & $21.4(14.5)$ & $25.6(13.4)$ & $27.0(14.0)$ \\
\hline & Total PA (counts $\times 10^{5} /$ week) & $34.3(6.3)$ & $33.9(7.8)$ & $33.6(7.3)$ & $32.0(7.1)$ \\
\hline & MVPA (min/day) & $44.5(15.5)$ & $43.7(18.0)$ & $41.9(16.9)$ & $37.6(13.8)$ \\
\hline
\end{tabular}

†Median (interquartile range).

BMI, body mass index; PA, physical activity; MV, moderate-and-vigorous; SD, standard deviation; WC, waist circumference.

Table 2 Correlations between total PA and BF\% (cross sectional and time lagged by $\pm 1,2$ and 3 years), partial $r(95 \% \mathrm{Cl})$

\begin{tabular}{lll}
\hline & \multicolumn{3}{l}{ Cross sectional (years) } \\
\cline { 2 - 3 } & TPA vs BF\% \\
\hline 7 vs 7 & $-0.22(-0.35 \text { to }-0.08)^{*}$ \\
8 vs 8 & $-0.20(-0.33 \text { to }-0.06)^{*}$ \\
9 vs 9 & $-0.18(-0.31 \text { to }-0.04)^{* *}$ \\
10 vs 10 & $-0.23(-0.36 \text { to }-0.09)^{* * *}$ \\
\hline Time-lagged (years) & TPA vs future BF\% & BF\% vs future TPA \\
\hline 7 vs 8 & $-0.17(-0.30 \text { to }-0.03)^{* *}$ & $-0.24(-0.37 \text { to }-0.10)^{* * *}$ \\
8 vs 9 & $-0.20(-0.33 \text { to }-0.06)^{*}$ & $-0.17(-0.30 \text { to }-0.03)^{* *}$ \\
9 vs 10 & $-0.12(-0.25$ to +0.02$)$ & $-0.26(-0.38 \text { to }-0.13)^{* * *}$ \\
7 vs 9 & $-0.14(-0.27 \text { to } 0.00)^{* *}$ & $-0.18(-0.31 \text { to }-0.04)^{* *}$ \\
8 vs 10 & $-0.17(-0.30 \text { to }-0.03)^{* *}$ & $-0.20(-0.33 \text { to }-0.06)^{*}$ \\
7 vs 10 & $-0.16(-0.29 \text { to }-0.02)^{* *}$ & $-0.21(-0.34 \text { to }-0.07)^{*}$ \\
\hline
\end{tabular}

All correlations in this table are partial correlations controlled for sex and age.

$\mathrm{n}=202$ for all analyses.

${ }^{*} p<0.05,{ }^{* *} p<0.01, * * * 0<0.001$.

$\mathrm{BF} \%$, body fat\%; TPA, total physical activity.

Table 4 Correlations of total PA versus change in $\mathrm{BF} \%$ and $\mathrm{BF} \%$ versus change in total PA, partial $r(95 \% \mathrm{CI})$

\begin{tabular}{lll}
\hline $\begin{array}{l}\text { Change in outcome } \\
\text { (years) }\end{array}$ & TPA vs change in BF\% & BF\% vs change in TPA \\
\hline 7 vs $\Delta 7-8$ & $+0.01(-0.13$ to +0.15$)$ & $-0.16(-0.29 \text { to }-0.02)^{*}$ \\
8 vs $\Delta 8-9$ & $-0.09(-0.23$ to +0.05$)$ & $-0.09(-0.23$ to +0.05$)$ \\
9 vs $\Delta 9-10$ & $+0.09(-0.05$ to +0.23$)$ & $-0.20(-0.33 \text { to }-0.06)^{* *}$ \\
7 vs $\Delta 7-9$ & $-0.02(-0.16$ to +0.12$)$ & $-0.07(-0.21$ to +0.07$)$ \\
8 vs $\Delta 8-10$ & $-0.06(-0.20$ to +0.08$)$ & $-0.10(-0.23$ to +0.04$)$ \\
7 vs $\Delta 7-10$ & $-0.05(-0.19$ to +0.09$)$ & $-0.13(-0.26$ to +0.01$)$ \\
\hline
\end{tabular}

All correlations in this table are partial correlations controlled for sex, age and the outcome variable obtained at the same time-point as the predictor variable. $\mathrm{n}=202$ for all analyses.

${ }^{*} \mathrm{p}<0.05$, ${ }^{*} \mathrm{p}<0.01$

$\Delta=$ change; $B F \%$, body fat $\%$; TPA, total physical activity.
Table 3 Correlations between MVPA and BF\% (cross sectional and time lagged by $\pm 1,2$ and 3 years), partial $r$ (95\% CI)

\begin{tabular}{lll}
\hline & \multicolumn{3}{l}{ Cross sectional (years) } \\
\cline { 2 - 3 } & MVPA vs BF\% \\
\hline 7 vs 7 & $-0.22(-0.35 \text { to }-0.08)^{* *}$ \\
8 vs 8 & $-0.23(-0.36 \text { to }-0.09)^{* * *}$ & \\
9 vs 9 & $-0.20(-0.33 \text { to }-0.06)^{* *}$ & \\
10 vs 10 & $-0.25(-0.38 \text { to }-0.12)^{* * *}$ & \\
\hline Time lagged (years) & MVPA vs future BF\% & BF\% vs future MVPA \\
\hline 7 vs 8 & $-0.16(-0.29 \text { to }-0.02)^{*}$ & $-0.31(-0.43 \text { to }-0.18)^{* * *}$ \\
8 vs 9 & $-0.23(-0.36 \text { to }-0.09)^{* * *}$ & $-0.21(-0.34 \text { to }-0.07)^{* *}$ \\
9 vs 10 & $-0.17(-0.30 \text { to }-0.03)^{*}$ & $-0.29(-0.41 \text { to }-0.16)^{* * *}$ \\
7 vs 9 & $-0.18(-0.31 \text { to }-0.04)^{*}$ & $-0.20(-0.33 \text { to }-0.06)^{* *}$ \\
8 vs 10 & $-0.23(-0.36 \text { to }-0.09)^{* *}$ & $-0.24(-0.37 \text { to }-0.10)^{* * *}$ \\
7 vs 10 & $-0.15(-0.28 \text { to }-0.01)^{*}$ & $-0.25(-0.38 \text { to }-0.12)^{* * *}$
\end{tabular}

All correlations in this table are partial correlations controlled for sex and age. $\mathrm{n}=202$ for all analyses.

${ }^{*} p<0.05,{ }^{* *} p<0.01, * * * 0<0.001$.

$\mathrm{BF} \%$, body fat $\%$; MVPA, moderate-and-vigorous physical activity.

Table 5 Correlations of MVPA versus change in $\mathrm{BF} \%$ and $\mathrm{BF} \%$ versus change in MVPA, partial $r$ ( $95 \% \mathrm{Cl}$ )

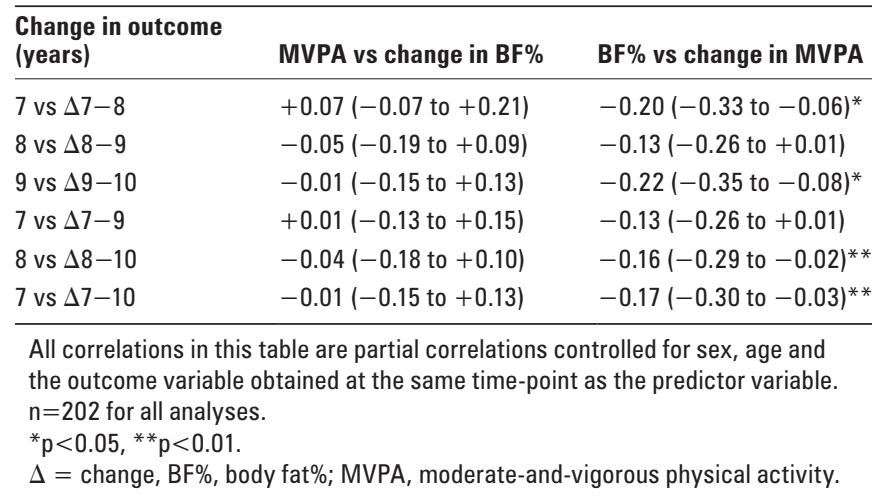


3 years ( $\mathrm{r}=-0.25 \mathrm{vs}-0.15)$ and were of similar strength to the cross-sectional correlations (table 2).

\section{Associations (change in outcome)}

There were no significant associations between PA and subsequent change in $\mathrm{BF} \%$, yet for the reverse analysis, $\mathrm{BF} \%$ versus changes in PA, half the associations were small to moderate, ranging from $\mathrm{r}=-0.16$ to -0.22 and statistically significant (tables 4 and 5). Using the longest follow-up period as an example, BF\% at 7 years predicted change in MVPA from 7 to 10 years $(r=-0.17, p=0.02)$, but MVPA at 7 years did not predict change in $\mathrm{BF} \%$ from 7 to 10 years $(r=-0.01, p=0.8)$. The slope coefficients obtained from the change in the MVPA model indicated that an additional $10 \% \mathrm{BF} \%$ at age 7 years results in a relative decrease in daily MVPA of 4 min from age 7 to 10 years $(p=0.02)$. The equivalent change in outcome associations that used BMI-SDS and WC-SDS in place of BF\% were weaker and not significant, although they were always slightly stronger for fatness predicting change in PA than for PA predicting change in fatness (eg, BMI-SDS 7 years vs $\triangle$ MVPA 7-10 years: $\mathrm{r}=-0.05, \mathrm{p}=0.2$; MVPA 7 years vs $\triangle \mathrm{BMI}-$ SDS 7-10 years: $r=-0.02, p=0.7$; WC-SDS 7 years vs $\triangle M V P A$ 7-10 years: $r=-0.07, p=0.2$; MVPA 7 years vs WC-SDS 7-10 years: $r=-0.02, p=0.7$ ).

\section{DISCUSSION}

\section{Principal findings}

This study confirms the inverse relationship between PA and $\mathrm{BF} \%$ reported previously by others. ${ }^{9}{ }^{10}$ It goes further, however, and suggests that this relationship is dominated by the impact of fatness on future activity rather than activity on future fatness. The data were consistent from year to year and in both sexes, and we believe that this is the first evidence to suggest direction of causality between fatness and activity in children.

\section{Strengths and weaknesses}

Ours is not an intervention study but uses time to imply the direction of causality. Importantly, the primary outcome measures were both obtained using objective techniques. Actigraph accelerometers and DEXA technology are considered criterion methods for measuring $\mathrm{PA}$ and $\mathrm{BF} \%$, respectively. $^{22} 24$ DEXA distinguishes fat from lean tissue and reports true adiposity (BF\%). BMI does not make the distinction, which is crucial because PA could readily increase lean/ muscle tissue. The lack of distinction between fat and lean may explain why the associations between PA and BMI in this study were weaker than those between PA and BF\%. The measurement of $\mathrm{BF} \%$ is a more precise measure than that of PA, and this could have contributed to our findings. When the less precise measure (PA) is the explanatory variable and the more precise measure (BF\%) is the outcome, the effect size could underestimate the true effect. The cohort represents an exclusively urban population $(98 \%$ of whom are Caucasian) from a single location, which may limit its generalisability. A final sample size of just $>200$ children may not be considered large by previous cross-sectional standards, but cross-sectional studies cannot address the question of causality. The present study was longitudinal, and the findings regarding causality are considered robust because they were shown to be consistent across six pairs of temporal relationships. Furthermore, although the final sample of $\mathrm{n}=202$ represents only $66 \%$ of the original cohort recruited at age 5 years, they were considered representative given that their BMI and PA levels at recruitment were no different from those of the 105 children excluded (BMI $p=0.4$, MVPA $\mathrm{p}=0.2$ ). Energy consumption is a notoriously unreliable measure in primary school children and could not be ruled out as a possible confounder. The study did, however, measure food choice by means of a food frequency questionnaire, but it had no impact on the PA versus fatness relationship and was not associated with either variable ( $p>0.5)$.

\section{Strengths and weaknesses in relation to other studies}

The literature contains a mix of studies addressing the relationship between PA and body mass. Some involve children, and others involve only adults. Some report BMI alone, and others report body composition, whereas some use subjective and others use objective measures to do so. Some are observational, and others are interventional. Crucially, some examine both directions of causality; others examine only one.

Four other longitudinal studies have exploited temporal relationships to examine causality in both directions between PA and body mass, but only in adults. ${ }^{12-15}$ All four found that a higher body mass/fat consistently increased the odds of becoming sedentary, whereas being sedentary rarely increased the odds of becoming obese. ${ }^{12-14}$ Although three of the four studies involved large sample sizes (ranging from 2500 to 5500) and long follow-up periods (10-25 years), all three nevertheless measured BMI rather than fatness and recorded PA by questionnaire, making it difficult to exclude reporting bias as a possible confounder. Ekelund and colleagues, ${ }^{15}$ on the other hand, recorded minute-byminute heart rate objectively over four consecutive days in 400 middle-aged adults. Body weight, BMI, girth and fat (by bio-impedance) all predicted sedentary time 6 years later (all $\mathrm{p} \leq 0.005$ ), whereas sedentary time did not predict future body weight, BMI, girth or fat (all p>0.05). Only one study has measured activity (accelerometry) and fatness (DEXA) at two time points in children. ${ }^{25}$ Riddoch and colleagues reported a small inverse association between activity at 12 years and fatness at 14 years but did not analyse the reverse association between fatness at 12 years and activity at 14 years and were, therefore, unable to determine the dominant direction of causality.

A recent meta-analysis of 12 randomised controlled trials ( $\mathrm{n}>8000$; average duration approximately 18 months) showed that school-based PA interventions had no impact on BMI (mean difference $0.01 \mathrm{~kg} / \mathrm{m}^{2} ; 95 \% \mathrm{CI}-0.14$ to 0.14 ) or $\mathrm{BF} \%{ }^{26}$ The authors concluded that there is evidence to suggest that reduced PA may be a downstream effect of obesity. There is only one experimental trial, however, that has measured the response of activity to change in weight. In a sample of 22 adults, Levine and colleagues ${ }^{27}$ showed that a gain of $2.8 \mathrm{~kg}$ in $\mathrm{BF} \%$ (achieved by an 8-week overfeeding intervention) decreased the distance walked by 1.5 miles/day $(p<0.001)$.

Wareham and colleagues ${ }^{11}$ reviewed 11 intervention studies that examined the response of body composition to PA (but not PA to body composition) in children. Benefit was reported in just three. ${ }^{11}$ Of those, only one could attribute the effect to exercise, as the interventions used in the other two involved diet and exercise combined. The authors commented that several of the trials that failed to show improvement in body composition nevertheless recorded measurable increases in PA. This was also the case in a recent randomised controlled trial involving 134 overweight preschool children in Glasgow, UK. ${ }^{28}$ The 6-month intervention increased levels of objectively 
measured total PA $(p<0.01)$ but had no impact on measures of body mass or girth (both $\mathrm{p} 0.4$ ).

Whereas neither the natural variation in PA among children nor attempts to increase the amount appear to influence the fatness of children, PA nevertheless has a demonstrable benefit on metabolic health. Our own published data suggest that $\mathrm{PA}$ at the government-recommended level is associated with dynamic improvements in metabolic health over time, even in the absence of a change in BMI or body composition. ${ }^{29} \mathrm{~A}$ further review by Wareham and colleagues ${ }^{30}$ reported on 13 PA-based interventions in children, 11 of which showed a reduction of insulin resistance levels.

\section{Meaning of the study}

There is no disputing the inverse association between PA and $\mathrm{BF} \%$. However, the present study suggests reverse causality as a plausible explanation for the failure of public health and school-based interventions promoting PA in children consistently to reduce BMI or other measures of $\mathrm{BF} \%$. Authors often speculate that their PA component was not sufficiently intense and intuitively recommend that future studies should provide more. Our findings suggest that rather than giving children ever-increasing doses of PA, we should first question the basic paradigm that more PA leads to less fat. If childhood fatness is not the result of physical inactivity, the implication may be that excess energy intake underlies fatness and inactivity.

\section{Possible explanations}

We do not know why fatter children should be less active, but psychological and physiological explanations appear to be plausible. A review by Sallis and colleagues ${ }^{31}$ showed that in three of seven studies, perceived body image was related to PA in adolescents. It is possible that overweight children perceive their body image negatively and, as a result, choose not to participate in sports and exercise. Several physiological reasons are discussed in a recent review by Shultz and colleagues. ${ }^{32}$ The authors suggest that exercise can cause musculoskeletal pain in overweight children. They imply that the extra energy costs of moving a greater body mass would cause breathlessness/fatigue sooner during exercise than for a normal-weight child. These symptoms may be due to the lower fitness of the fatter child or associated with mitochondrial dysfunction. There is emerging evidence to suggest that excess $\mathrm{BF} \%$ and the insulin resistance associated with it may lead to mitochondrial dysfunction. ${ }^{33}$ Mitochondria are responsible for delivering aerobic energy, crucial to muscle fitness. Failure to supply energy aerobically at the rate it is needed leads to the accumulation of lactate, causing muscle discomfort and limiting tolerance to intense muscular effort.

\section{Unanswered questions and future research}

Further research is required in children to test this reverse causality more formally with experimental trials. If our findings are replicated, future research should aim to provide a better understanding of why fatness leads to inactivity but inactivity does not lead to fatness. It is still not clear what type of intervention is the most likely to succeed in preventing childhood obesity. However, given the relative success that dietary interventions have had in improving BMI, ${ }^{34}$ future intervention studies should, perhaps, focus more on reducing energy intake than on increasing energy expenditure.

\section{CONCLUSIONS}

The image of the "couch-potato" child who is obese because he is sedentary runs deep in Western consciousness. However, the possibility that the reverse obtains, that his fatness is the cause rather than the result of his inactivity, has far-reaching implications. Although there may be many benefits to PA, the findings of this study, coupled with the limited success of PA interventions aimed at improving BMI, imply that public health strategies may need to target energy intake to curb the year-on-year rise in childhood obesity.

Acknowledgements The authors thank Karen Brookes and Val Morgan for their assistance with the data collection and Sharan Griffin for operating the DEXA machine.

Funding This report from the EarlyBird Diabetes Study was funded by the Bright Futures Trust, Diabetes UK, Smith's Charity, the Child Growth Foundation, the Diabetes Foundation, the Beatrice Laing Trust, Abbott, Astra-Zeneca, GSK, Ipsen and Roche. None of the sources funding this study had any involvement in the study design, collection or analysis of data, interpretation of findings or writing of the manuscript.

\section{Competing interests None.}

Contributors The authors have contributed the following and have seen and approved the final version of this article. BSM, child health statistician: data entry, analysis and writing. $\mathrm{JH}$, child health statistician: data entry and analysis. ANJ, research nurse: data collection. LDV, coordinator of the EarlyBird study: writing. WH, University lecturer in statistics: statistical advice and analysis. TJW, director of the EarlyBird study: writing.

Ethical approval Ethical approval was obtained from the Plymouth local research ethics committee (South and West Devon Health Authority) in 1999. Informed consent and assent were obtained from parents and children, respectively.

Provenance and peer review Not commissioned; externally peer reviewed.

\section{REFERENCES}

1. Galal $\mathbf{O M}$, Hulett J. Obesity among schoolchildren in developing countries. Food Nutr Bull 2005;26:S261-6.

2. Department of Health. Obesity among children under 11-health survey for England 1995-2003. 2007. http://www.dh.gov.uk/en/Publicationsandstatistics/ Publications/PublicationsStatistics/DH_4109245 (accessed Dec 2009).

3. Ogden CL, Carroll MD, Curtin LR, et al. Prevalence of overweight and obesity in the United States, 1999-2004. JAMA 2006;295:1549-55.

4. Weiss R, Caprio S. The metabolic consequences of childhood obesity. Best Pract Res Clin Endocrinol Metab 2005;19:405-19.

5. ADA. Type 2 diabetes in children and adolescents. American Diabetes Association. Diabetes Care 2000;23:381-9

6. Ball EJ, O'Connor J, Abbott R, et al. Total energy expenditure, body fatness, and physical activity in children aged 6-9 y. Am J Clin Nutr 2001;74:524-8.

7. National Institute for Health and Clinical Excellence (NICE). Department of Health. Promoting physical activity, active play and sport for pre-school and school-age children and young people in family, pre-school, school and community settings. 2009. http://www.nice.org.uk/nicemedia/pdf/PH017Guidance.pdf (accessed Dec 2009).

8. Department of Health. Change4Life. 2009. http://www.nhs.uk/change4life/ Pages/Default.aspx (accessed Dec 2009).

9. Ekelund U, Yngve A, Brage S, et al. Body movement and physical activity energy expenditure in children and adolescents: how to adjust for differences in body size and age. Am J Clin Nutr 2004;79:851-6.

10. Ness AR, Leary SD, Mattocks C, et al. Objectively measured physical activity and fat mass in a large cohort of children. PLOS Med 2007;4:e97.

11. Wareham NJ, van Sluijs EM, Ekelund U. Physical activity and obesity prevention: a review of the current evidence. Proc Nutr Soc 2005;64:229-47.

12. Bak H, Petersen L, Sørensen TI. Physical activity in relation to development and maintenance of obesity in men with and without juvenile onset obesity. Int $\mathrm{J}$ Obes Relat Metab Disord 2004;28:99-104.

13. Petersen L, Schnohr P, Sørensen TI. Longitudinal study of the long-term relation between physical activity and obesity in adults. Int J Obes Relat Metab Disord 2004;28:105-12

14. Mortensen LH, Siegler IC, Barefoot JC, et al. Prospective associations between sedentary lifestyle and BMI in midlife. Obesity (Silver Spring) 2006;14:1462-71.

15. Ekelund $\mathbf{U}$, Brage S, Besson $\mathrm{H}$, et al. Time spent being sedentary and weight gain in healthy adults: reverse or bidirectional causality? Am J Clin Nutr 2008;88:612-7.

16. Office of the Deputy Prime Minister. State of the English cities_chapter 5: social cohesion in English cities. Vol 1. 2006:109-54. 
http://webarchive.nationalarchives.gov.uk/+/http://www.communities.gov.uk/pub/4/ StateoftheEnglishCitiesVolume1PDF33Mb_id1164004.pdf (accessed Jun 2010).

17. Voss LD, Kirkby J, Metcalf BS, et al. Preventable factors in childhood that lead to insulin resistance, diabetes mellitus and the metabolic syndrome: the EarlyBird diabetes study 1. J Pediatr Endocrinol Metab 2003;16:1211-24.

18. Puyau MR, Adolph AL, Vohra FA, et al. Validation and calibration of physical activity monitors in children. Obes Res 2002;10:150-7.

19. Schmitz KH, Treuth M, Hannan P, et al. Predicting energy expenditure from accelerometry counts in adolescent girls. Med Sci Sports Exerc 2005;37:155-61.

20. Metcalf BS, Curnow JS, Evans $\mathrm{C}$, et al. Technical reliability of the CSA activity monitor: the EarlyBird study. Med Sci Sports Exerc 2002;34:1533-7.

21. Thorsen S. Weather in UK-England-Plymouth time and date. 1995-2008. http://www.timeanddate.com/weather/uk/plymouth (accessed 6 Nov 2008)

22. Lohman TG, Chen Z. Dual energy $x$-ray absorptiometry. In: Heymsfield SB, Lohman TG, Wang Z, Going SB, eds. Human body composition. 2nd edn. Champaign, Illinois, USA: Human Kinetics, 2005:63-77.

23. Twisk JWR. Other possibilities for modelling longitudinal data. In: Applied longitudinal data analysis for epidemiology: a practical guide. Cambridge: Cambridge University Press, 2003:102-19.

24. Reilly JJ, Penpraze V, Hislop J, et al. Objective measurement of physical activity and sedentary behaviour: review with new data. Arch Dis Child 2008;93:614-9.

25. Riddoch CJ, Leary SD, Ness AR, et al. Prospective associations between objective measures of physical activity and fat mass in 12-14 year old children: the Avon Longitudinal Study of Parents and Children (ALSPAC). BMJ 2009:339:b4544.
26. Harris KC, Kuramoto LK, Schulzer M, et al. Effect of school-based physical activity interventions on body mass index in children: a meta-analysis. CMAJ 2009;180:719-26.

27. Levine JA, McCrady SK, Lanningham-Foster LM, et al. The role of free-living daily walking in human weight gain and obesity. Diabetes

2008; 57:548-54.

28. Hughes AR, Stewart L, Chapple J, et al. Randomized, controlled trial of a best-practice individualized behavioral program for treatment of childhood overweight: Scottish Childhood Overweight Treatment Trial (SCOTT). Pediatrics 2008; 121:e539-46.

29. Metcalf BS, Voss LD, Hosking J, et al. Physical activity at the governmentrecommended level and obesity-related health outcomes: a longitudinal study (EarlyBird 37). Arch Dis Child 2008;93:772-7.

30. Wareham NJ, Brage S, Franks PW, et al. Relationship between physical activity and insulin resistance. In: Kumar S, O'Rahilly S. Insulin resistance: insulin action and its disturbances in disease. Chichester: Wiley, 2004:317-400.

31. Sallis JF, Prochaska JJ, Taylor WC. A review of correlates of physical activity of children and adolescents. Med Sci Sports Exerc 2000;32:963-75.

32. Shultz SP, Anner J, Hills AP. Paediatric obesity, physical activity and the musculoskeletal system. Obes Rev 2009;10:576-82.

33. Szendroedi J, Roden M. Mitochondrial fitness and insulin sensitivity in humans. Diabetologia 2008;51:2155-67.

34. Collins CE, Warren J, Neve M, et al. Measuring effectiveness of dietetic interventions in child obesity: a systematic review of randomized trials. Arch Pediatr Adolesc Med 2006:160:906-22. 